\title{
Preparing Nurses to Fight Covid-19 and Similar Calamities
}

\author{
Ukande $\mathrm{UM}^{*}$ \\ Choithram College of Nursing, India
}

*Corresponding author: Usha Mulllick Ukande, Choithram College of Nursing, 303, Sky Star Apt. N-1 New Rani Bag, Indore Pin 452020 MP, India, Tel: +91 9425438183; Email: usha_mullick@ yahoo.com

Corona pandemic has shaken the world to its core. Most of the nurses and doctors were thrown into the pandemic situation rather unprepared. There was not enough time to train them adequately. Second problem faced by almost all over the world was not enough PPE kits. Other preparations too were a big challenge: not enough hospital beds and/or to have fully dedicated hospitals for corona positive patients. Then if you convert super-specialty hospitals into Red category only for corona cases because these hospitals had the facilities for critical case management for corona positive patients who would require continuous oxygen supply and ventilators. Then what about those other patients who were getting specialized services in these few fully equipped tertiary care hospitals. Truly, many patients such as those requiring regular haemodialysis, Thalassemia children needing regular blood transfusions and others who needed emergency services for conditions of heart, brain etc. These patients suffered till some other arrangements were done for them.

While the whole world was in the middle of this chaos, nurses had proved the most confident HCW because they had already learned about basic hygienic practices, knew about principles of quarantine and isolation, disinfection techniques, logistic management and universal standard precautions. However, they too experienced a number of inconveniences in the beginning when they had to face this challenge almost unprepared for this huge pandemic. The long working hours of 10-12 in full PPEs, without taking food or drink and not even going to the wash room. And then if they were coming back to their own home after duty, they were afraid of infecting their family members especially the children. Nurses were getting exhausted physically as well as emotionally, seeing many patients dying in spite of the best care they received but without specific cure against this deadly virus. This added to the psychological stress the nurse was already exposed to. The burn out among nurses took its toll and many got infected with covid-19 and had to be admitted in the hospital for treatment. Some nurses and doctors succumbed to corona. Nurses got so scared that they decided to quit nursing practice and many actually did this. This further created shortage of nurses and those who were working; their work-load increased making them more vulnerable. WHO and governments prepared and circulated fresh guidelines based on the available experiences and data, this helped the policy makers to plan strategies which made work place facilities better including the availability of adequate equipment and supplies. Now, the situation was a little more favourable for the nurses and doctors to work in hospitals dedicated for corona positive patients.

All this has led us to think how best to prepare our nurses for a situation like this in the future. Nurses form the largest task force of the healthcare manpower and are responsible for delivering direct care to the patients in the hospitals and people in the community. In times of natural or man-made disasters, their role becomes more important. Therefore, preparing them to face such challenges confidently and efficiently should be started from the time an individual is selected for becoming a nurse at the entry point itself. Choice of taking up nursing as a career should be made after having a deep understanding of what nursing really means. Individuals can seek advice from the career counselling experts or someone who has successfully completed nursing course and is practicing as a nurse. Once you have entered in to this stream, it is the work of the mentors and others who help in transforming a raw young person in to a competent, efficient and compassionate nurse and it is no doubt a tremendous and challenging one, as the nursing education is called 'maturity in the making'.

Now, preparing nurses for challenges like this pandemic, we need to further equip them with certain tools during the period of internship and while they are practicing nursing in the clinical field. The tools are in the form of periodical and planned in-service education programs to regularly enhance their knowledge and strengthen their competencies. This will lead them to: 
- Know and practice established occupational safety protocols.

- Participate in occupational safety and health trainings.

- Use provided protocols to assess triage and treat patients.

- Not to underestimate any disaster.

- Keep them fully appraised with latest authentic evidence based data.

- Keeping calm and to carry on.

However, throughout the process of preparing the nurses for any unexpected situation like the present pandemic, need is to create a balance in discipline of nursing practice, nursing profession and nursing informatics. The Nursing profession, in recent years, with rise of external crises of economics, management science, technology, too much medicalized hospital-based practices and policies, has been detoured from its disciplinary foundation. Therefore, the basic concern of discipline of nursing, the humanistic approach should remain in every nurse's mind at all times, and also they should not get lost in the flood of information available on the internet but to be selective in gaining and using the information.

Let us now look at what good has been brought by this pandemic situation. It has opened new doors of opportunities to the nurses for the future:
- The field of nursing informatics will be explored more for application.

- Nurses now will be seeking to use telehealth and virtual care for patients.

- They will have more leadership opportunities in hospital care delivery systems.

- Explore new possibilities in research.

- They will be takingactive role in patient care management and decision making in hospitals and community health care settings.

- More nurses will choose to specialize and in new areas like 'nursing informatics'

- Nurses will ask for better funding for continued education and up-skilling in digital literacy.

- A new approach and framework will be developed for nursing practice

Challenge that covid-19 pandemic posed for the world, has been faced by the nurses bravely and together with doctors they were designated as 'corona warriors'. yes, they are our soldiers fighting at the frontline and for them to continue serving bravely they too need to keep themselves fit. Here are a few suggestions for our nurses to keep their physical and mental health intact: Exercise, take up time to relax, meditate and have a healthy diet, ensure breaks and adequate sleep, practice relaxation exercises like yoga and meditation, stay connected-even when physically isolated. Take good care of yourselves.

- Dr Usha Mullick Ukande , Director Care Continuum, India 